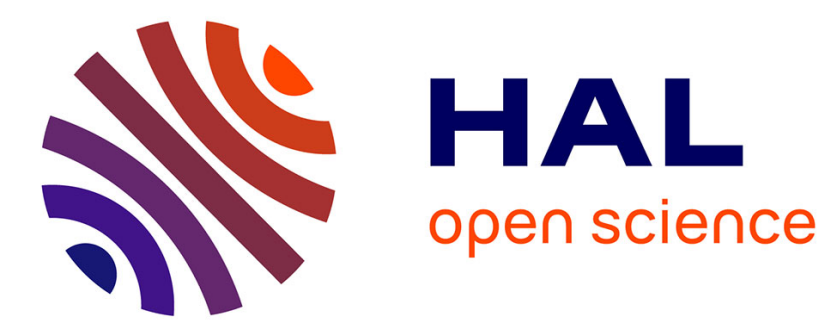

\title{
Faraday waves on band pattern under zero gravity conditions
}

Tatyana Lyubimiva, Andrey Ivantsov, Yves Garrabos, Carole Lecoutre, Daniel Beysens

\section{- To cite this version:}

Tatyana Lyubimiva, Andrey Ivantsov, Yves Garrabos, Carole Lecoutre, Daniel Beysens. Faraday waves on band pattern under zero gravity conditions. Physical Review Fluids, 2019, 4 (6), 064001 (16 p.). 10.1103/PhysRevFluids.4.064001 . hal-02150260

\section{HAL Id: hal-02150260 \\ https://hal.science/hal-02150260}

Submitted on 29 Jul 2020

HAL is a multi-disciplinary open access archive for the deposit and dissemination of scientific research documents, whether they are published or not. The documents may come from teaching and research institutions in France or abroad, or from public or private research centers.
L'archive ouverte pluridisciplinaire HAL, est destinée au dépôt et à la diffusion de documents scientifiques de niveau recherche, publiés ou non, émanant des établissements d'enseignement et de recherche français ou étrangers, des laboratoires publics ou privés. 


\title{
Faraday waves on band pattern under zero gravity conditions
}

\author{
T. Lyubimova, ${ }^{1,2}$ A. Ivantsov, ${ }^{1,2}$ Y. Garrabos, ${ }^{3,4}$ C. Lecoutre, ${ }^{3,4}$ and D. Beysens ${ }^{5,6}$ \\ ${ }^{1}$ Institute of Continuous Media Mechanics of the Ural Branch, RAS, 1, Koroleva Street, 614013, Perm, Russia \\ ${ }^{2}$ Perm State University, 15, Bukireva Street 614990, Perm, Russia \\ ${ }^{3}$ CNRS, ICMCB, ESEME, UMR 5026, F-33600 Pessac, France \\ ${ }^{4}$ Université Bordeaux, ICMCB, UMR 5026, F-33600 Pessac, France \\ ${ }^{5}$ Service des Basses Températures, CEA-Grenoble \& Université Joseph Fourier, Grenoble, France \\ ${ }^{6}$ Physique et Mécanique des Milieux Hétérogènes, CNRS PSL-ESPCI Sorbonne Université Paris Cité, \\ 10 Rue Vauquelin, 75005, Paris, France
}

(Received 31 July 2018; published 3 June 2019)

\begin{abstract}
Observations performed with $\mathrm{CO}_{2}$ near its critical point onboard sounding rockets show that periodical patterns (bands) develop in a two-phase, liquid-vapor system under tangential vibrations in microgravity conditions. Fluids are slightly below their liquid-vapor critical point where liquid and vapor densities are close, surface tension is low, and they exhibit a scaled, universal behavior. With the increase of vibration amplitude, an instability can develop on the band pattern, leading to the appearance of Faraday waves. Theoretical and numerical investigations of the Faraday instability onset and development are carried out taking into account the interaction between the bands. The critical parameters for the onset of instability are determined. The comparisons between theoretical analysis, two-dimensional direct numerical simulation, and original experimental data show good agreement.
\end{abstract}

DOI: 10.1103/PhysRevFluids.4.064001

\section{INTRODUCTION AND BACKGROUND}

In the space environment, gravity effects are not present and a number of phenomena, of marginal effect on earth, can become predominant. This is particularly the case for a number of fluid instabilities triggered by vibrations when the stabilizing effect of gravity is not present any more. It is of both industrial and academic interest to investigate such instabilities. The management of fluids in space is indeed one of the major concerns for engineers, and unveiling new fluid processes is one of the most exciting finding for scientists.

When subjected to vibration, the shape and stability of the interface between two fluids depend on the relative direction of the vibration and the interface. It is known that in a gravity field a quasistationary wave pattern (frozen wave instability [1]) can develop at the interface when the applied vibration is parallel to the initially horizontal fluid interface. In Ref. [2] this phenomenon was studied for the case of two superposed infinite horizontal layers of inviscid fluids. The stability of the base state with a flat interface parallel to the layer boundaries and plane-parallel timeperiodical longitudinal flows in both layers was considered [Fig. 1(a)]. Because of the difference in densities of the fluids, the base flow velocities in the two layers are different; thus a tangential velocity jump across the interface appears, leading to a shear-driven Kelvin-Helmholtz (KH) instability. If the layer thicknesses are not too small, a finite-wavelength instability develops with the increase of vibration intensity [Fig. 1(b)]. The interface is immobile ("frozen") in average in the reference frame of the oscillating boundaries.

The theory developed by Lyubimov and Cherepanov [2] for the description of this phenomenon assumes that the pulsational flow is irrotational and incompressible. In the "high" frequency limit 

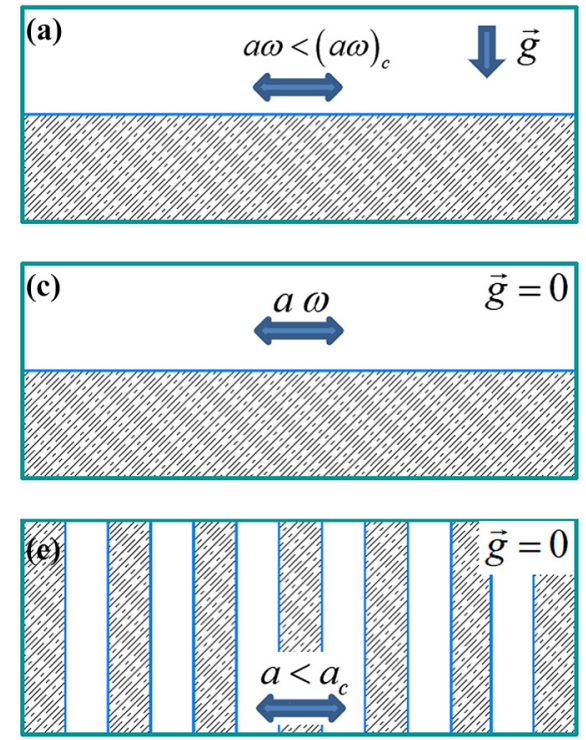
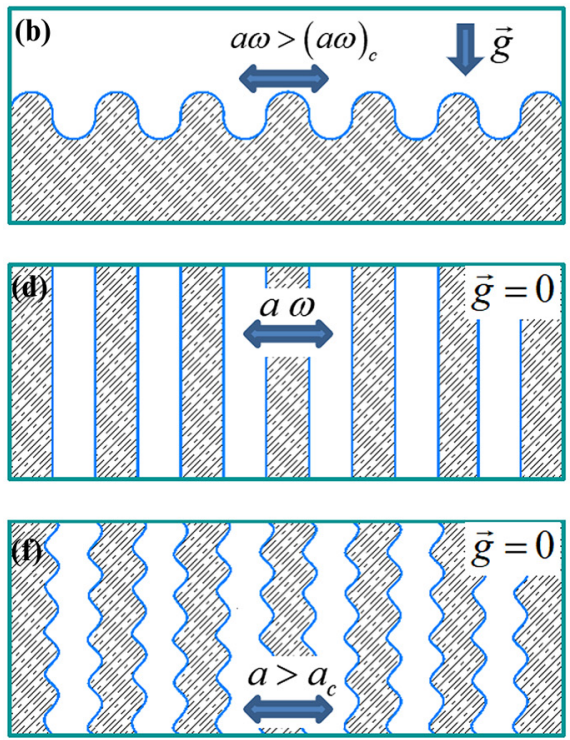

FIG. 1. Interface between two fluids: (a), (b) in a gravity field, and (c)-(f) in zero gravity conditions; (a), (c), (e) base states, (b), (d), (f) supercritical regimes: (b) frozen waves at $a \omega>(a \omega)_{c}$, (d) band pattern, (f) Faraday waves on bands at $a>a_{c}$.

$\omega \gg v / L^{2}(\omega=2 \pi f$ is the vibration angular frequency; $f$ is frequency; $v=\eta / \rho$ is the kinematic viscosity of the two fluids, assumed equal; and $L$ is a typical length scale) and small vibration amplitude $a \ll L$, the slow motion of the interface can be decoupled from its fast motion. Using this decomposition and performing a linear stability analysis the critical conditions for the onset of instability in an infinitely long horizontal layer with flow closeness condition were predicted in [2] in the form

$$
(a \omega)^{2} \geqslant \frac{1}{2}(a \omega)_{c}^{2}\left(\frac{\lambda}{\lambda_{0}}+\frac{\lambda_{0}}{\lambda}\right) \tanh \left(\frac{2 \pi h}{\lambda}\right) .
$$

Here

$$
(a \omega)_{c}^{2}=\frac{\left(\rho_{1}+\rho_{2}\right)^{3}}{\rho_{1} \rho_{2}\left(\rho_{2}-\rho_{1}\right)} \sqrt{\frac{\sigma g}{\rho_{2}-\rho_{1}}}
$$

is the critical value of the squared vibration velocity amplitude at the instability onset, $h$ is the thickness of one liquid layer, $\lambda$ is the perturbation wavelength, and

$$
\lambda_{0}=2 \pi l_{c}
$$

is the critical wavelength, with

$$
l_{c}=\sqrt{\frac{\sigma}{g\left(\rho_{2}-\rho_{1}\right)}},
$$

the capillary length (here $\sigma$ is the surface tension coefficient; $g$ is the acceleration of gravity). When the vibration velocity amplitude is critical, $a \omega=(a \omega)_{c}$ and the wavelength of the quasistationary profile is equal to $\lambda_{0}[2]$.

The expression of the instability condition Eq. (1) is similar to the condition obtained from a linear stability analysis of the classical Kelvin-Helmholtz instability. Equation (2) predicts that the 
critical vibration velocity amplitude diverges when $\rho_{2} / \rho_{1}$ is large, leading to a stable interface. That is why this instability is only observed for fluids of comparable densities [2-4] like liquid and vapor phases near a critical point [5].

It follows from a weakly nonlinear analysis of the instability near the threshold [2] that the bifurcation can be subcritical or supercritical depending on the parameters. For a supercritical bifurcation the expression for the amplitude $A$ of the frozen wave has the form (within the assumption $\lambda<h$, i.e., $\tanh (2 \pi h / \lambda) \approx 1)$

$$
A=\lambda_{0} \frac{2\left(\rho_{1}+\rho_{2}\right)^{2}}{\pi \rho_{1}^{2} \sqrt{-11\left(\rho_{2} / \rho_{1}\right)^{2}+42 \rho_{2} / \rho_{1}-11}} \Delta(a \omega)^{*},
$$

where

$$
\Delta(a \omega)^{*}=\frac{\sqrt{(a \omega)^{2}-(a \omega)_{c}^{2}}}{(a \omega)_{c}}
$$

is the normalized distance to the critical vibration velocity amplitude.

Note that the theoretical results (1)-(4) were obtained for a system of infinite layers. Nevertheless, numerical modeling [6] and experiments [7,8] performed in closed containers gave results that well agree with the stability condition (1).

Under zero $g$, when the stabilizing effect of gravity is absent, the amplitude of the frozen waves thus diverges, forming bands oriented perpendicularly to the vibration direction [see Figs. 1(c) and 1(d)] as observed in $\mathrm{CO}_{2}$ [5,9,10], $\mathrm{H}_{2}$ [11,12], liquid mixtures of FC-40 and silicone oil [13], and miscible mixtures of water-isopropanol with water [14]. This orienting effect of vibrations was predicted theoretically in [15]. Although both frozen wave and band instabilities have the same origin (shear flow at the interface) the band characteristics cannot be simply deduced from a mere extrapolation at $g=0$ of the frozen wave instability, where both amplitude $A$ and wavelength $\lambda_{0} \sim l_{c}$ diverge. In the case of an infinitely long layer the long-wave perturbations are the most dangerous and the instability threshold is zero for these perturbations. For any nonzero value of the vibration velocity amplitude the perturbations with the wave number grow from a certain range [see the neutral curve $B(k)$ (interrupted curve) in Fig. 2]. The question on the selection of critical perturbations as realized in experiments therefore arises. As shown in [5] by a linear stability analysis, in this case the band periodicity is defined by the wave number of the perturbations with maximal growth rate. An analytical solution describing the dependence of the wave number of perturbations with maximal growth rate on the vibration velocity amplitude for inviscid fluids has the form [5]

$$
(a \omega)^{2}=\frac{\left(\rho_{1}+\rho_{2}\right)^{3}}{4 \rho_{1} \rho_{2}\left(\rho_{2}-\rho_{1}\right)^{2}} \sigma k\left[3 \tanh (k h)+\frac{k h}{\cosh ^{2}(k h)}\right] .
$$

The dependence of the wave number of perturbations with maximal growth rate on the dimensionless vibration parameter is plotted in Fig. 2 (full curve). For viscous fluids it is not possible to obtain analytical expressions similar to Eq. (7). The problem was solved numerically by the solution of a linearized problem on the evolution of small perturbations of the base state [5]. It was found that the band wave number strongly depends on viscosity.

Thus, according to (1) the critical value of the vibration velocity amplitude tends to zero when gravity vanishes; i.e., in the case of zero gravity, a KH instability could take place at any values of $a \omega$. However, recent calculations carried out for containers of finite length [13] have demonstrated the existence of a nonzero-critical vibration intensity. These results are not in contradiction with the zero-critical vibration as reported in [5], where, for infinite layers, the long wave perturbations are the most dangerous and the critical value of $a \omega$ for these perturbations is equal to zero. In a container of finite length $L$ the perturbation wavelength is limited by $L$ and the minimal possible wave number is $2 \pi / L$, which corresponds to a nonzero value of $a \omega$ (see Fig. 2). The same conclusions are valid 


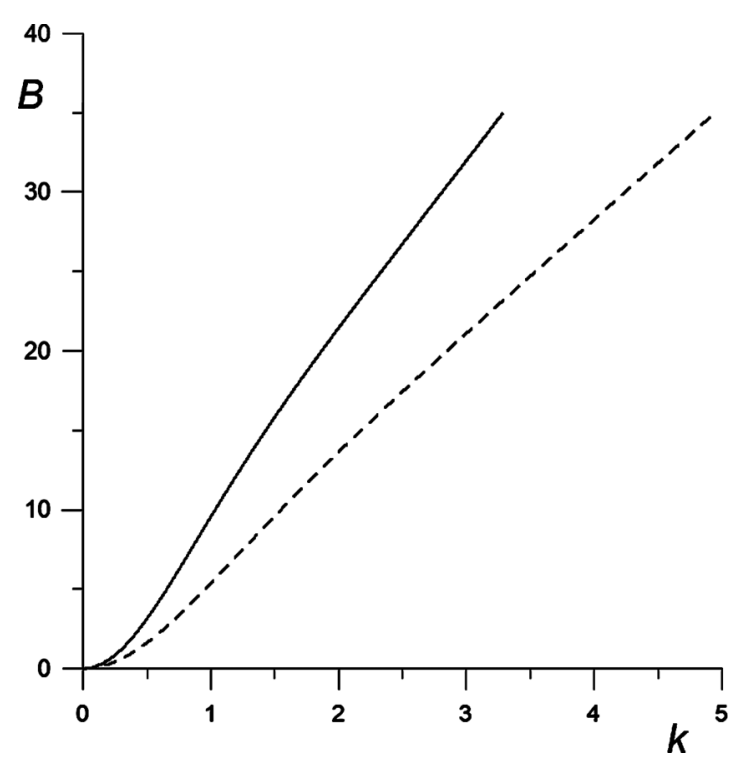

FIG. 2. Dependence of the dimensionless vibrational parameter $B=(a \omega)^{2} h\left(\rho_{2}-\rho_{1}\right) / 4 \sigma$ on the dimensionless wave number $k$ for $\rho=1.15$ (taken from [5]). Interrupted curve: neutral perturbations with $\lambda=0$ in an inviscid fluid; full curve: perturbations with maximal growth rate in an inviscid fluid.

for the perturbations with maximal growth rate which, as shown in [5], are responsible for the KH instability in zero gravity conditions.

When the band pattern is established, the fluid interface becomes perpendicular to the vibration direction and one naturally expects the development of a Faraday wave instability when the vibration intensity exceeds a critical value [see Figs. 1(e) and 1(f)]. Faraday instabilities have indeed been observed at the liquid-vapor interface in $\mathrm{H}_{2}$ near its critical point [12] and at the interface of miscible water-alcohol mixtures [14].

The goal of the present paper is to analyze these Faraday waves on band patterns, analytically, numerically, and by processing experimental data obtained for $\mathrm{CO}_{2}$ under microgravity conditions in the sounding rocket Maxus 7. As mentioned above, the two-phase system used in our experiments is characterized by a low surface tension. In this case, the values of the dimensionless vibration parameter $B$ are large and, as can be seen from Fig. 2, the dimensionless wave numbers of perturbations with maximal growth rate are also large. Thus, the wavelength of the most dangerous perturbations is small; i.e., formation of thin bands is expected. Therefore, differing from the previous studies, the interaction between the interfaces should be important, which can substantially modify the instability characteristics. In the present paper we thus study the excitation of Faraday waves on the band pattern by taking into account this unique particular situation and compare with original data obtained under zero gravity conditions.

The paper is organized as follows. The next section is devoted to an experimental section with methods and observations. Direct numerical simulation and theoretical analysis of the Faraday waves' instability onset are afterward reported and their results are compared with the experimental observations.

\section{EXPERIMENTAL OBSERVATION OF FARADAY WAVES IN MICROGRAVITY}

\section{A. Fluids near their critical point}

The use of fluids in the vicinity of their liquid-vapor critical point is particularly appealing to carry out such investigations. In addition to its use by the space industry in such near supercritical 
TABLE I. Parameters of $\mathrm{CO}_{2}$ and $n-\mathrm{H}_{2}$ [17].

\begin{tabular}{lcccccc}
\hline \hline Fluid & $\begin{array}{c}T_{c} \\
(\mathrm{~K})\end{array}$ & $\begin{array}{c}p_{c} \\
(\mathrm{MPa})\end{array}$ & $\begin{array}{c}\rho_{c} \\
\left(\mathrm{~kg} \mathrm{~m}^{-3}\right)\end{array}$ & $\beta$ & $\begin{array}{c}\sigma_{0} \\
\left(10^{-2} \mathrm{~N} \mathrm{~m}^{-1}\right)\end{array}$ & $\begin{array}{c}\eta \\
\left(10^{-5} \mathrm{~Pa} \mathrm{~s}\right)\end{array}$ \\
\hline $\mathrm{CO}_{2}$ & 304.14 & 7.375 & 467.8 & 1.60 & 6.72 & 4.21 \\
$n-\mathrm{H}_{2}$ & 33.19 & 1.315 & 30.11 & 1.61 & 0.542 & 0.27 \\
\hline \hline
\end{tabular}

conditions, the thermodynamic properties of fluids when expressed under reduced temperature or density distance from the critical point can be conveyed under universal, scaled functions [16]. Using such scaling, studying one fluid permits us to study all. In addition, the fact that surface tension $\sigma$ and liquid-vapor density difference $\Delta \rho$ vanish when nearing the critical point enhances the effect of accelerations and highlights the mechanical instabilities. Experimentally speaking, another interest of considering the critical point vicinity is the possibility to vary the fluid parameters simply by changing temperature.

A number of fluid properties can be described in the vicinity of the critical point by scaling laws [16]. The densities of vapor $\left(\rho_{v}\right)$ and liquid $\left(\rho_{l}\right)$ phases behave asymptotically (with $\rho_{c}$ the critical density) according to

$$
\rho_{l, v}=\rho_{c}\left(1 \pm \beta \varepsilon^{0.325}\right)
$$

Here

$$
\varepsilon=\left|\frac{T-T_{c}}{T_{c}}\right|,
$$

with $T$ as temperature and $T_{c}$ the critical temperature. The value of parameter $\beta$ depends on the considered fluid. The density difference $\Delta \rho=\rho_{l}-\rho_{v}$ follows

$$
\Delta \rho=2 \beta \rho_{c} \varepsilon^{0.325},
$$

and the mean density (rectilinear diameter with negligible slope) is

$$
\frac{\rho_{l}+\rho_{v}}{2}=\rho_{c}
$$

The liquid-vapor interfacial tension obeys

$$
\sigma=\sigma_{0} \varepsilon^{1.26},
$$

where $\sigma_{0}$ is a fluid-dependent parameter. The viscosities of both phases are close to each other and do not appreciably change when nearing the critical point.

\section{B. Experimental setup}

Experiments have been performed with $\mathrm{CO}_{2}$ onboard sounding rockets. The physical parameters of $\mathrm{CO}_{2}$ are listed in Table I together with the critical parameters ( $p_{c}$ is the critical pressure, and $\eta$ is the dynamic viscosity of the fluid).

Details on the experimental setup can be found in [5,10]. A sketch is given in Fig. 3. The experimental module is of Texus Experiment Module by Ferrari (TEM-FER) type [10]. The latter is an $\mathrm{Al}$ cylinder in which is inserted the cell made of copper-cobalt-beryllium alloy. It is thermally regulated by a Peltier element within $0.3 \mathrm{mK}$ accuracy. Temperature can be adjusted within 1 $\mathrm{mK}$ steps. As the module has to work under vacuum during the flight, the thermostat remains pressurized at 1 bar pressure. 


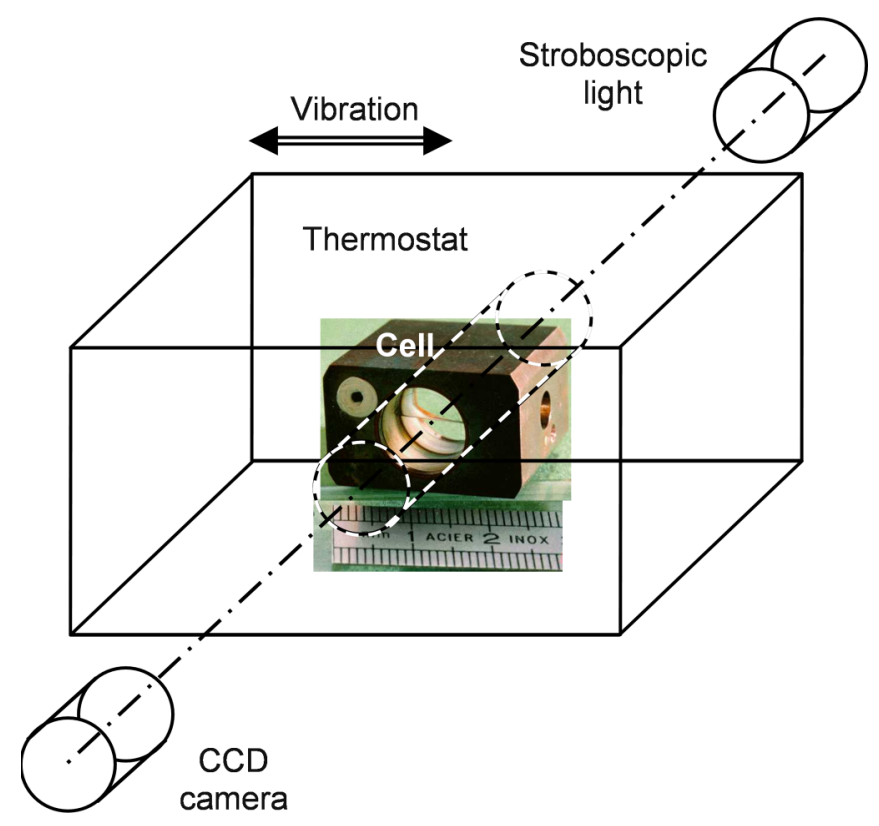

FIG. 3. Experimental arrangement of the $\mathrm{CO}_{2}$ experiment (schematic). The double arrow indicates the vibration direction. The scale is in centimeters.

In the cell body is drilled a cylinder with radius $R=5.0 \mathrm{~mm}$ and thickness $e=2.189 \mathrm{~mm}$, closed at each end by two parallel sapphire windows. Using $\mathrm{CuCoBe}$ alloy ensures good thermal conduction between the fluid and the thermostat. The sample is illuminated by parallel light issued from a light-emitting diode (LED) and observed in transmission by two cameras. Coherent illumination is used to enhance the refractive-index gradients and then the density gradients. The cell is filled at critical density with a precision of $0.1 \%$, by checking the temperature variation of the meniscus position according to the method developed in [18].

The sample cell is inserted into one arm of a two-arms symmetric shaker vibrating device. In order to prevent vibrations of the rocket, another block (hosting experiments with vibrated granular materials) with the same mass is vibrated in the opposite arm, in phase opposition. The couple of the motor is also compensated to prevent rocket rotation. The shaker can apply linear harmonic vibrations in a range of amplitude $a=0.2-2.5 \mathrm{~mm}$ and frequency $f=0.5-50.5 \mathrm{~Hz} ; a$ and $f$ can be varied independently step by step. A three-axis accelerometer measures the accelerations; a linear variable-differential transformer measures the amplitude of the alternative motion of the thermostat in the vibration direction.

Images are taken by a high-resolution, $2048 \times 2048$ pixels, 5 fps frame rate, $125 \mu$ s full-frame shutter times, fixed gain, digital camera. It is equipped with a long-working-distance microscope. Images are stored on the rocket module and recovered after the flight. With an imaging factor of $1: 1.12$, the final spatial resolution is on the order of 1 pixel $(7.4 \mu \mathrm{m} \times 7.4 \mu \mathrm{m})$. Image analysis is then used to determine the characteristics of the pattern. All other scientific data, including images with low resolution $(782 \times 582$ pixels $)$ from a $25 \mathrm{~Hz}$ charge-coupled device $(\mathrm{CCD})$ camera, temperature measurements, vibration amplitude and frequency, and acceleration data, are sent by telemetry to the ground.

Timeline (Table II) is the following. A first set of experiments was dedicated to phase transition from an initial state at $2 \mathrm{mK}$ above $T_{c}$. Equilibration under weightlessness was ensured until $t=75 \mathrm{~s}$ after lift-off (time $t=0$ by definition). Then vibration $(0.31 \mathrm{~mm}, 20.2 \mathrm{~Hz})$ is applied. A temperature quench of $4.4 \mathrm{mK}$ is performed at $t=150 \mathrm{~s}$. The cell is maintained at the same temperature until the end of the period allocated for the experiment $(t=539 \mathrm{~s})$ where the liquid and vapor phases 
TABLE II. Timeline of the $\mathrm{CO}_{2}$ weightlessness experiment. MET: Mission elapsed time; $a$ and $f$ are measured quantities.

\begin{tabular}{|c|c|c|c|c|}
\hline $\operatorname{MET}(\mathrm{s})$ & $T-T_{c}(\mathrm{mk})$ & $a(\mathrm{~mm})$ & $f\left(\mathrm{H}_{\mathrm{z}}\right)$ & Action \\
\hline-10800 & 300 & & & 1-h equilibration period \\
\hline-7200 & $300-2.0$ & & & Stepping down in log steps \\
\hline-1200 & 2.0 & & & Equilibration \\
\hline 0 & 2.0 & & & Rocket lift-off \\
\hline 75 & 2.0 & & & Start of $\mu \mathrm{g}$ period and fluid relaxation \\
\hline 135 & 2.0 & 0.305 & 20.3 & Start of vibration $(0.31 \mathrm{~mm}, 20.2 \mathrm{~Hz})$ \\
\hline 150 & -2.4 & 0.48 & 25.25 & Quench-down and relaxation \\
\hline 539 & -2.4 & 1.41 & 25.25 & New input of vibration parameters \\
\hline 567 & -2.4 & 2.5 & 20.3 & \\
\hline 593 & -2.4 & 2.5 & 10.5 & \\
\hline 624 & -2.4 & 2.5 & 5.6 & \\
\hline 659 & -2.4 & 1.0 & 5.6 & \\
\hline 679 & -2.4 & 0.20 & 5.6 & \\
\hline 711 & -2.4 & 0.70 & 10.5 & \\
\hline 730 & -2.4 & 0.70 & 25.2 & \\
\hline 761 & -2.4 & 0.20 & 25.2 & \\
\hline 786 & -2.4 & 0.20 & 49.7 & \\
\hline 818 & -2.4 & 0.71 & 49.7 & \\
\hline $843-854$ & -2.4 & 0.21 & 0 & End \\
\hline
\end{tabular}

appear as well organized bands perpendicular to the vibration direction [10]. Then amplitude and vibration are varied about each $30 \mathrm{~s}$ until the end of weightlessness $(800 \mathrm{~s})$, thus corresponding to 13 different combinations $(a, f)$. The time to change $(a, f)$ is about $2 \mathrm{~s}$.

Concerning high-resolution pictures, which are taken at a $5 \mathrm{fps}$ frame rate $(0.2 \mathrm{~s}$ time period $)$ and $125 \mu \mathrm{s}$ full-frame shutter times, the frame rate is lower than the vibration frequency. It corresponds to taking successive images every $0.2 \mathrm{~s}$ at times $t_{0}=0$ and $t_{1}=n T+1 \times \Delta t=0.2 \mathrm{~s}, t_{2}=n T+$ $2 \times \Delta t=0.4 \mathrm{~s}$, etc. $t_{p}=n T+(p \times \Delta t=p \times 0.2 \mathrm{~s})$, with $n$ and $p$ integer numbers and $\Delta t$ the time lag determined by $\Delta t=(0.2 / T)-n$. It means that it will need $p=T / \Delta t$ images to describe the equivalent of one period.

\section{Observation}

A number of interesting instabilities is then evidenced under vibrations. Typically, the fluid (here $\mathrm{CO}_{2}$ ) is subjected to harmonic vibrations and the relative distance to the critical temperature $\epsilon=$ $\left(T_{c}-T\right) / T_{c}$ is varied. The liquid-vapor interface is seen to successively exhibit the following five stages as $\sigma$ and $\Delta \rho$ diminish with $\varepsilon$. (i) $\varepsilon>3.3 \times 10^{-3}$ : The vapor bubble oscillates, with a number of modes that increases when $\varepsilon$ decreases [19]; (ii) $10^{-3}<\varepsilon<3.3 \times 10^{-3}$ : The bubble shows a flat interface perpendicular to vibrations [19,20]; (iii) $3 \times 10^{-4}<\varepsilon<10^{-3}$ : The Faraday waves (square pattern) develop on the liquid-vapor interface, whose wavelength decreases when $\varepsilon$ decreases [12]; (iv) $7 \times 10^{-5}<\varepsilon<2 \times 10^{-4}$ : The Faraday waves square pattern becomes a two-dimensional (2D) line pattern due to small wavelength increased dissipation [12,21]; (v) $\varepsilon<7 \times 10^{-5}$ : The liquidvapor phases order in parallel "bands" perpendicular to the vibration direction [Fig. 1(d)]. These bands are reminiscent of the earth-bound frozen wave instability where the liquid-vapor interface deformation is immobile in the reference frame of the oscillating boundaries [5]. The bands can be seen as frozen wave amplitudes much larger than the sample size [see Figs. 1(b) and 1(d)].

In the band regime (v), Faraday waves can be observed on the band interfaces (which are perpendicular to the vibration direction) [5]. The Faraday instability corresponds to the Faraday roll pattern on the single liquid-vapor interface as observed in [12]. There is, however, a noticeable difference as the band thickness is much smaller than the sample size. Such band pattern has also 


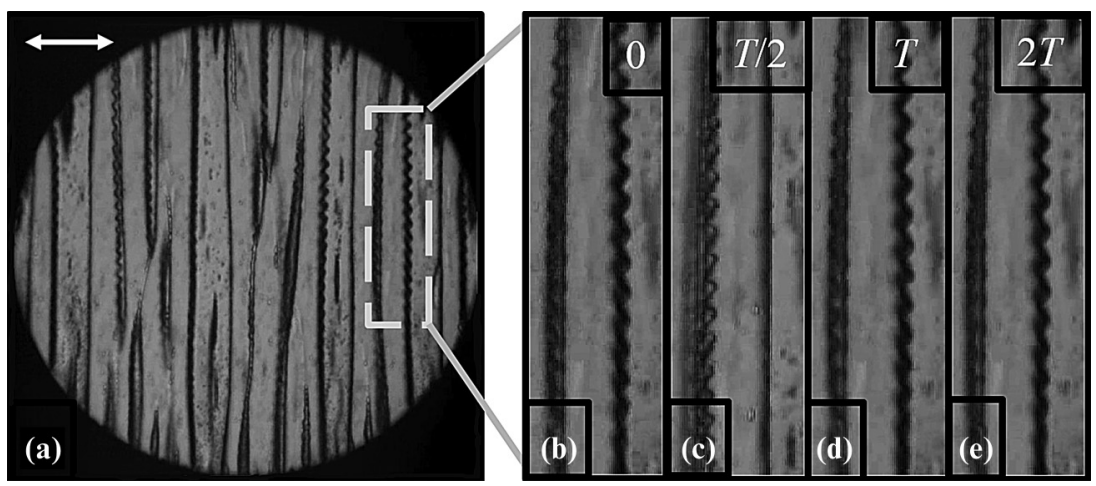

FIG. 4. (a) $\mathrm{CO}_{2}$ sample ( $10.0 \mathrm{~mm}$ diameter and $2.189 \mathrm{~mm}$ thickness) under $a=0.7 \mathrm{~mm}, f=25.25 \mathrm{~Hz}$ vibrations with direction indicated by a double arrow. The window is enlarged in (b)-(e). The black lines correspond to the interfaces between the liquid and vapor phases, which order as "bands" perpendicular to the vibration direction. (b)-(e) Different times in units of vibration period $T$ (see text) showing subharmonicity by the interface deformation phase as highlighted by the white dotted line). Time origin corresponds to amplitude maximum on the right. Interface deformation occurs on a half period on one side of the band (b), (d), (e) and on the other half period on the other side (c).

been noted under weightlessness with miscible liquid mixtures just after they were put into contact, and the same kind of Faraday waves were also observed [14].

A typical band pattern is reported in Fig. 4 under $a=0.7 \mathrm{~mm}, f=25.25 \mathrm{~Hz}$ vibrations, near the instability threshold. Interfaces are seen to exhibit line deformations that correspond to Faraday waves. As a matter of fact, the subharmonic character of the interface instability can be characterized by the fact that the phase of the interface deformation is recovered after twice the excitation period [Figs. 4(b), 4(d), and 4(e)].

Concerning fluids near a critical point, the same phenomenon as noted above in Sec. IIC occurs: As the wavelength of the instability decreases when approaching the critical point, enhanced dissipation induces a transition from square pattern to line pattern. This transition depends on both $T_{c}-T$ (which determines the pattern wavelength $\lambda$ ) and viscous dissipation $\left[\eta(a \omega)^{2} / f \lambda^{2}\right]$, at a temperature sufficiently away from $T_{c}$ where the fluid does not yet order into bands (see Sec. II B). Transition square line occurs typically within about $T_{c}-T \approx 10 \mathrm{mK}$ in $\mathrm{H}_{2}$ [12].

\section{NUMERICAL SIMULATIONS}

\section{A. Methology}

The numerical simulations were performed for a system of two immiscible fluids filling a rectangular container of length $l$ and height $2 h$. A Cartesian coordinate system was chosen with the axis $z$ directed vertically upwards and axis $x$ directed along the layer. The container is subjected to vibrations at amplitude $a$ and frequency $\omega$.

The initial state corresponds to the band structures with interfaces perpendicular to the vibration axis. The period of the structure is made either regular, corresponding to the analysis performed in [5], or slightly varied. In the latter case, the initial structure was obtained by the additional calculation of the bands' formation process from an initially flat fluid interface under tangential vibrations.

In the reference frame of an oscillating container, the system of equations is written as follows:

$$
\begin{aligned}
\rho_{s}\left(\frac{\partial \overrightarrow{\mathrm{v}}_{s}}{\partial t}+\overrightarrow{\mathrm{v}}_{s} \cdot \nabla \overrightarrow{\mathrm{v}}_{s}\right)= & -\nabla p_{s}+\eta_{s} \Delta \overrightarrow{\mathrm{v}}_{s}+\rho_{s} a \omega^{2} \vec{e}_{x} \cos \omega t \\
& \operatorname{div} \overrightarrow{\mathrm{v}}_{s}=0
\end{aligned}
$$


where the subscript $s=1,2$ denotes the fluids, $\overrightarrow{\mathrm{v}}_{s}$ is the fluid velocity, $p_{s}$ is the pressure, $\rho_{s}$ is the density, $\eta_{s}$ is the dynamic viscosity, and $\vec{e}_{z}$ are the basis vectors of the coordinate system. At the interfaces the usual conditions for normal and tangential stress balance, velocity continuity, and kinematic condition are imposed:

$$
\begin{gathered}
\left(p_{2}-p_{1}\right) \vec{n}=\left(\bar{\tau}^{(2)}-\bar{\tau}^{(1)}\right) \cdot \vec{n}+\sigma \vec{n} \operatorname{div} \vec{n}, \\
\overrightarrow{\mathrm{v}}_{1}=\overrightarrow{\mathrm{v}}_{2}, \\
\frac{\partial \zeta}{\partial t}+\overrightarrow{\mathrm{v}}_{1} \cdot \nabla \zeta=\overrightarrow{\mathrm{v}}_{1} \cdot \vec{e}_{z} .
\end{gathered}
$$

Here $\vec{n}$ is the unit vector normal to the interface and directed toward the less dense fluid, $\bar{\tau}^{s}=$ $\tau_{i k}^{s}=\eta_{s}\left(\partial \mathrm{v}_{s, i} / \partial x_{k}+\partial \mathrm{v}_{s, k} / \partial x_{i}\right)$ is the viscous stress tensor, and $\sigma$ is the surface tension coefficient. The no-slip condition is imposed at the container walls.

The modeling of the interface dynamics under horizontal vibrations is carried out by direct numerical simulations. The calculations are performed using the volume of fluid (VoF) method for the description of the dynamics of the interface of immiscible fluids. This method is based on the introduction of the volume fraction of each phase, $\psi_{s}$, which is constant inside a selected phase and sharply changes at the interface between two phases. The variables and properties in any point are defined by the values of volume fractions. Therefore, a multiphase system is treated as a single medium with the parameters sharply changing at the interfaces, and the Navier-Stokes equations describing the system can be solved in the same way all over the computational domain with an additional source term in the momentum equation (13) describing the surface tension. The great advantage of this approach is that there is no need to deal with the boundary conditions (15)-(17) at the interface. The VoF method is implemented in the ANSYS FLUENT software, which was used in [6] to model the frozen wave formation.

Tracking of the interface between the two phases is accomplished by the solution of the continuity equation for the volume fraction of the phases. For the $s$ phase, this equation has the following form:

$$
\frac{\partial \psi_{s}}{\partial t}+\overrightarrow{\mathrm{v}}_{s} \cdot \nabla \psi_{s}=0
$$

The volume fraction of the primary phase is determined from the condition

$$
\psi_{1}+\psi_{2}=1
$$

The influence of vibrations is taken into account by introducing a periodically varying volumetric force. The surface tension can be expressed as the volume force in the momentum equation (13):

$$
F_{\mathrm{vol}}=\sigma \frac{\rho \kappa_{s} \nabla \psi_{s}}{\bar{\rho}},
$$

where $\bar{\rho}$ is the mean density, $\bar{\rho}=\left(\rho_{1}+\rho_{2}\right) / 2 ; \rho$ is the density in the computational cell given by the expression $\rho=\psi_{1} \rho_{1}+\psi_{2} \rho_{2} ; \kappa_{s}=\nabla \cdot \vec{n}_{s} /\left|\vec{n}_{s}\right|$ is the interface curvature defined in terms of the divergence of the unit vector $\vec{n}_{s}$ : The spatial discretization is performed using the third-order MUSCL scheme (monotone upstream-centered schemes for conservation laws). At each time step the convergence criterion for the velocity components is used. The absolute scaled residual errors have to be less than 0.001 ("scaled" means that the residual error is divided by the flow rate through the domain).

\section{B. Simulation for $\mathrm{CO}_{2}$ experiment}

The $\mathrm{CO}_{2}$ parameters (Table I) are used at temperature $T-T_{c}=-2.4 \mathrm{mK}$ (Table II). Most of the simulations are carried out for a rectangular container of $2 h=1.2 \mathrm{~mm}$ height and $l=8 \mathrm{~mm}$ 


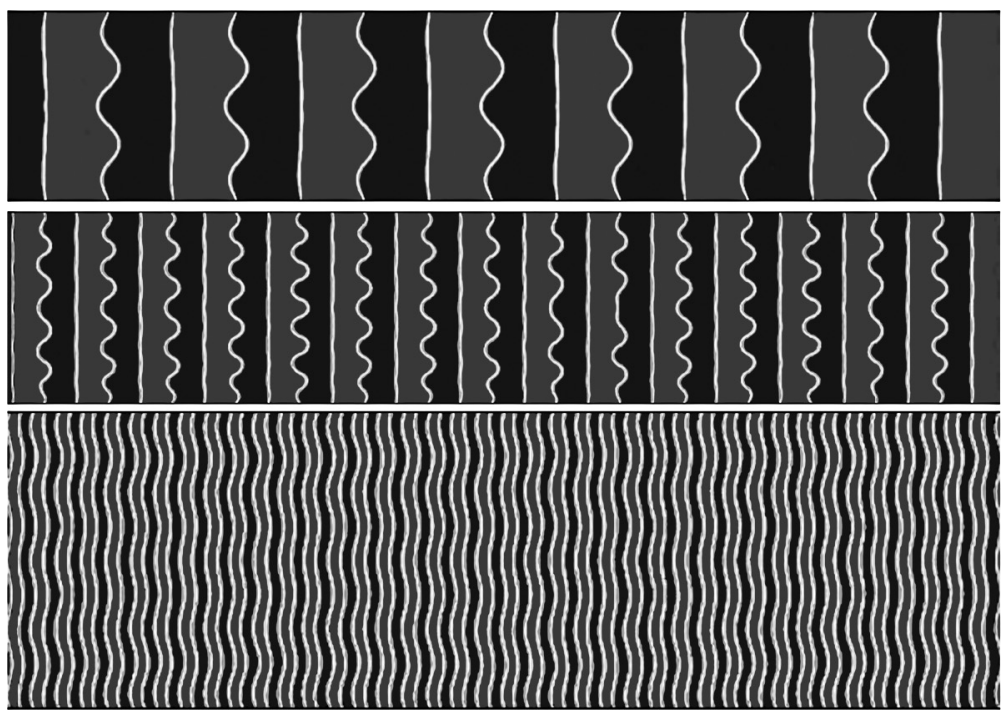

(a)

(b)

(c)

FIG. 5. Faraday waves for different parameters of vibrations: (a) $f=5 \mathrm{~Hz}, a=2.5 \mathrm{~mm}$, band pattern period $=0.8 \mathrm{~mm}$; (b) $f=25 \mathrm{~Hz}, a=1.1 \mathrm{~mm}$, band pattern period $=0.4 \mathrm{~mm}$; (c) $f=20 \mathrm{~Hz}, a=1.6 \mathrm{~mm}$, band pattern period $=0.1 \mathrm{~mm}$.

long. In these simulations the initial state corresponds to the state with a constant band pattern period [Fig. 1(e)]. The simulations show that the dependence of the Faraday wave characteristics and instability threshold on the band period is not very strong (see Fig. 5). Significant stabilization of the system (growth of the critical vibration amplitude) is observed for small values of the band period, smaller than the Faraday wavelength.

The case where the band pattern period is much smaller than the wavelength of the Faraday waves gives rise to a mode with synchronous deviations of all fluid interfaces [zero phase shift; see Fig. 5(c)]. It is very likely that the wall effect is the main reason for its appearance. Therefore, this mode should be difficult to observe in experiments and we do not discuss it further in this paper.

\section{Fourier analysis of Faraday waves}

To assess that the instability studied experimentally and numerically in the previous sections is indeed a Faraday instability, Fourier analyses of the velocity in the central point of the cavity were performed. As one can see in Fig. 6, the leading frequency $(2.5 \mathrm{~Hz})$ of oscillations is twice as small as the frequency $(5 \mathrm{~Hz})$ of external vibrations (note that the peak at $7.5 \mathrm{~Hz}$ corresponds to the second Fourier harmonic and the peak at $12.5 \mathrm{~Hz}$ to the third). As a consequence, the waves on the bands indeed correspond to subharmonic mode of oscillations; i.e., the waves are similar to classic Faraday waves.

In Fig. 7 the band patterns are shown for different phases of the vibration period. One can notice that the phase shift of nearby interfaces is $1 / 4$ of the Faraday wave period (or $1 / 8$ of the vibration period). This phase shift was observed in all calculations except for the case of a very small period of band patterns when, as is mentioned above, another mode with zero phase shift arises.

\section{THRESHOLD OF FARADAY WAVES ON BANDS}

\section{A. Theoretical analysis of Faraday wave instability threshold}

The experiments and the theory described above show that, in the weightlessness of the space environment, a two-layer system under tangential vibrations forms a band pattern with the interfaces 


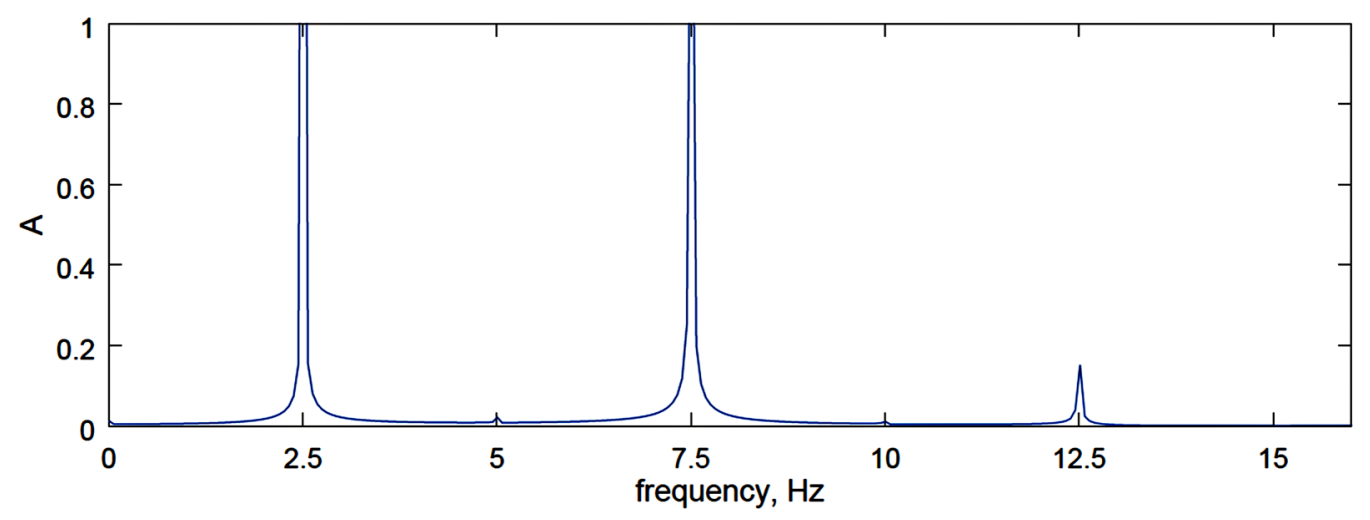

FIG. 6. Fourier analysis of velocity evolution in the central point of the cavity for frequency $f=5 \mathrm{~Hz}$ and $a=2.5 \mathrm{~mm}$; $A$ (in arbitrary units) is the amplitude of the Fourier component.

perpendicular to the vibration direction. At high enough vibration amplitudes, the Faraday wave instability develops at the interfaces. In this section we analyze the critical conditions for this instability using the approach of Kumar and Tuckerman [22], taking into account low viscosity of fluids.

We consider a band pattern formed in the system of layers of immiscible fluids, in a large container that corresponds to the structure observed in experiments. Fluid interfaces are perpendicular to the direction of vibrations and the layers are of equal depth (Fig. 8). In this section, the Cartesian coordinate system with the axis $z$ directed along the vibration axis is used. Near the stability threshold the solution could be assumed periodic in the direction of the $z$ axis. It is therefore sufficient to consider the two-layer system with the conditions of periodicity shown by the interrupted line in Fig. 8.

In the reference frame of an oscillating container, the dynamics of the system is described by the equations and boundary conditions Eqs. (13)-(17), completed with the condition of periodicity imposed at $z=+h,-h$. The solution of the problem that corresponds to the base state with flat interfaces reads

$$
\overrightarrow{\mathrm{v}}_{\mathrm{s}}^{0}=0, \quad p^{0}=\rho_{s} a \omega^{2} \cos (\omega t) z
$$

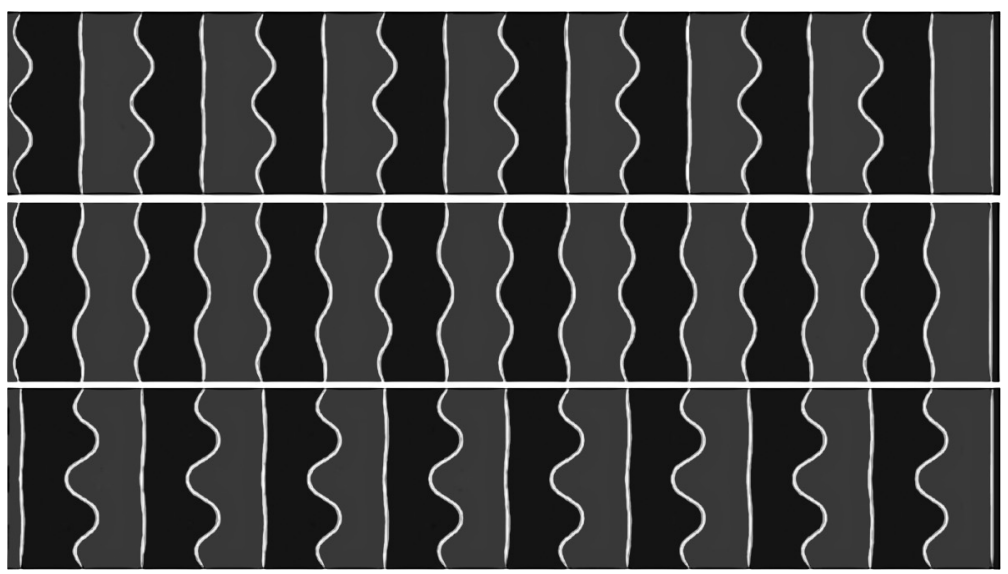

(a)

(b)

(c)

FIG. 7. Band patterns for different phases of Faraday waves period for $f=5 \mathrm{~Hz}, a=2.5 \mathrm{~mm}$, band period $=0.8 \mathrm{~mm}$ : (a) $\varphi=0$, (b) $\varphi=\pi / 8$, (c) $\varphi=\pi / 4$. 


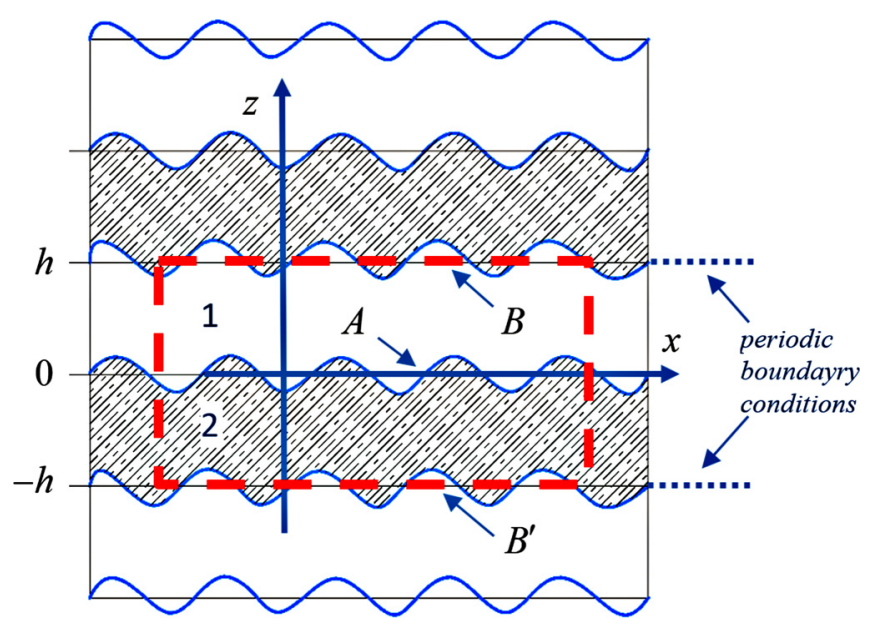

FIG. 8. Scheme of linear stability analysis of Faraday waves on bands.

Linearizing the governing equations and implementing the operation of $\nabla \times \nabla \times$, we obtain the equations for the linear stability problem [22],

$$
\frac{\partial \nabla^{2} w_{s}}{\partial t}-v_{s} \nabla^{2} \nabla^{2} w_{s}=0,
$$

where $w_{s}$ is the perturbation of the vertical velocity components. As was mentioned (see also Table I), the viscosity of the considered fluids is quite small. Based on that, we completely neglect fluid viscosity in a first step. This assumption reduces the problem to a system of two Mathieu equations. However, as in [22], viscosity is taken into account in a phenomenological way by introducing an additional dissipation term in the Mathieu equations. We also neglect surface tension since it is vanishing in fluids near their critical point [Eq. (5)].

According to the Floquet theorem, the solution could be presented in the following form:

$$
w_{j}=\sum_{n=-\infty}^{n=\infty} w_{j n}(z) e^{i n \omega t} .
$$

In the case of inviscid fluids Eq. (22) can be written as

$$
\nabla^{2} w_{s}=0
$$

and the boundary conditions (15)-(17) for perturbations are

$$
\begin{gathered}
w_{1}=w_{2}, \\
\left(\rho_{2}-\rho_{1}\right) \frac{\partial^{2}}{\partial t \partial z} w=-\left[\left(\rho_{2}-\rho_{1}\right) a \cos (\omega t)\right] k^{2} \zeta, \\
\frac{\partial \zeta_{s}}{\partial t}=w_{s} .
\end{gathered}
$$

Solutions to Eq. (24) that satisfy the boundary condition (25) at both interfaces and the conditions of periodicity read

$$
\begin{aligned}
& w_{1}=\left[\tilde{a}(t) e^{-k z}+\tilde{b}(t) e^{-k(h-z)}\right] e^{i k x}, \\
& w_{2}=\left[\tilde{a}(t) e^{k z}+\tilde{b}(t) e^{-k(h+z)}\right] e^{i k x},
\end{aligned}
$$


where $\tilde{a}(t), \tilde{b}(t)$ are time-dependent amplitudes. Note that, according to the condition of periodicity at the second boundary $B, B^{\prime}$ (see Fig. 8), it is necessary to use $z=h$ for $w_{1}$, and $z=-h$ for $w_{2}$. Then, from the kinematic condition Eq. (27), one obtains for the first interface (marked $A$ in Fig. 8)

$$
\dot{\zeta}_{A}=\tilde{a}(t)+\tilde{b}(t) e^{-k h}
$$

and for the second interface $\left(B, B^{\prime}\right.$ in Fig. 8)

$$
\dot{\zeta}_{B}=\tilde{a}(t) e^{-k h}+\tilde{b}(t)
$$

Substituting (28)-(31) in the dynamic boundary condition Eq. (26) we obtain the system of two Mathieu equations describing the behavior of the interfaces,

$$
\begin{aligned}
& \ddot{\zeta}_{A} \operatorname{coth}(k h)-\ddot{\zeta}_{B} \operatorname{csch}(k h)+2 \gamma \dot{\zeta}_{A}+F \cos (\omega t) \dot{\zeta}_{A}=0, \\
& \ddot{\zeta}_{B} \operatorname{coth}(k h)+\ddot{\zeta}_{A} \operatorname{csch}(k h)+2 \gamma \dot{\zeta}_{B}-F \cos (\omega t) \dot{\zeta}_{B}=0,
\end{aligned}
$$

where

$$
F=\frac{a \omega^{2}\left(\rho_{2}-\rho_{1}\right) k}{\rho_{1}+\rho_{2}}, \quad \gamma=\frac{2 k^{2}\left(\eta_{1}+\eta_{2}\right)}{\rho_{1}+\rho_{2}}
$$

and $\operatorname{coth}(x)=\cosh (x) / \sinh (x)$ is hyperbolic cotangent; $\operatorname{csch}(x)=1 / \sinh (x)$ is hyperbolic cosecant.

In order to obtain analytical expressions, we use the simplification suggested in [14] where the solution was approximated by using only two Fourier components $\zeta=\zeta_{+} e^{i \omega t / 2}+\zeta_{-} e^{-i \omega t / 2}$. As is mentioned in Sec. IV B in numerical computation only the mode with the quarter period shift in phases of neighboring interface oscillations,

$$
\zeta_{1}(t)=\zeta_{2}\left(t+\frac{\pi}{4}\right)
$$

was observed, therefore we restrict ourselves by consideration of this mode. Additionally, the amplitudes of interface deviations $\left(\zeta_{1}, \zeta_{2}\right)$ are assumed to be the same, which also corresponds to the numerical and experimental observations. Taking into account the above assumptions and the smallness of the viscosity, we obtain the solution to Eqs. (32) and (33) in the form

$$
(a \omega)^{2}=\frac{\left(\omega^{2}+4 \gamma^{2}+8 \omega \gamma e^{-k h}+6 \omega^{2} e^{-2 k h}\right)\left(\rho_{1}+\rho_{2}\right)^{2}}{\left(1-e^{-2 k h}\right)^{2}\left(\rho_{2}-\rho_{1}\right)^{2} k^{2}} .
$$

Minimizing the neutral curve Eq. (35) with respect to the wave number, we obtain

$$
a_{c}=\frac{2 \sqrt{2 e^{-2 H}+\sqrt{6 e^{-2 H}+1}}}{\left(\rho_{2}-\rho_{1}\right)\left(1-e^{-2 H}\right)} \sqrt{\frac{\left(\rho_{2}+\rho_{1}\right)\left(\eta_{1}+\eta_{2}\right)}{\omega}},
$$

where $H=k h$ is the dimensionless spatial period of the band pattern. In the case of large $H$, when interaction of interfaces is negligible, this formula is reduced to the estimate of the critical amplitude obtained in [14] for a single fluid interface:

$$
a_{c}=\frac{2}{\rho_{2}-\rho_{1}} \sqrt{\frac{\left(\rho_{2}+\rho_{1}\right)\left(\eta_{1}+\eta_{2}\right)}{\omega}} .
$$

\section{B. Comparison of numerical and experimental results}

The formula, Eq. (36), for the threshold of the Faraday waves on bands shows that the critical vibration amplitude is subjected to a sharp growth in the range of a small period of bands. This result well corresponds qualitatively to the numerical modeling (Fig. 9). When compared to numerical 


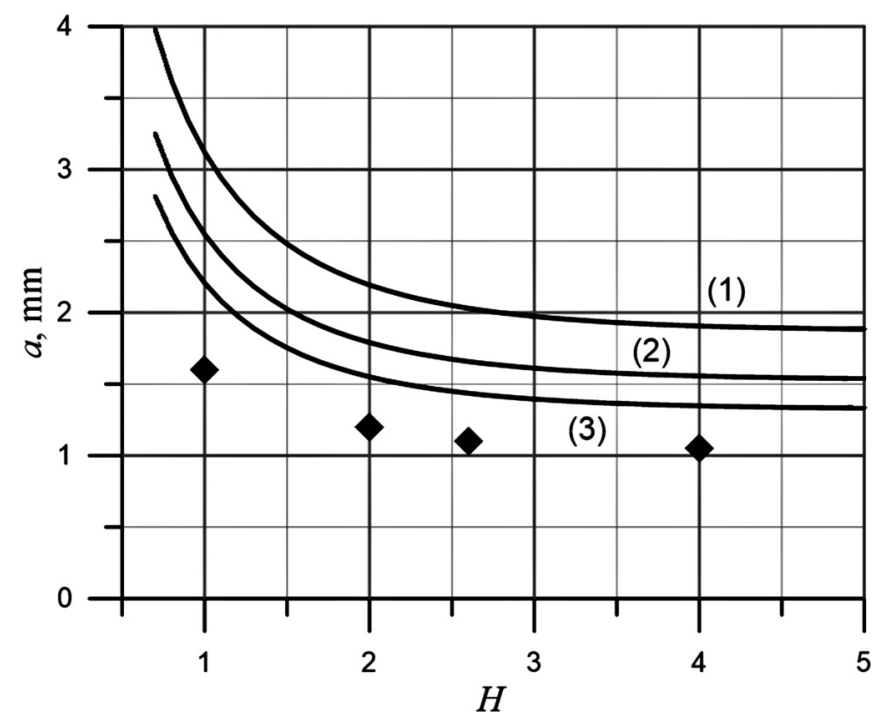

FIG. 9. The dependences of critical vibration amplitude on the dimensionless layer depth $H$ for (1) $f=$ $10 \mathrm{~Hz}$, (2) $f=15 \mathrm{~Hz}$, (3) $f=20 \mathrm{~Hz}$ according to Eq. (36). The parameters correspond to $\mathrm{CO}_{2}$ experiments, see Table I. Diamonds correspond to the stability boundary obtained in the numerical simulations for $f=20 \mathrm{~Hz}$.

simulations, the overestimation of the critical amplitude given by Eq. (36) is expected since similar deviation was found in [14] for Eq. (37) corresponding to a single fluid interface.

Figure 10 shows the comparison of analytical results for the critical vibration amplitude with the stability curves obtained in the direct numerical simulation of Sec. IV B and experiments. The latter are in good agreement.

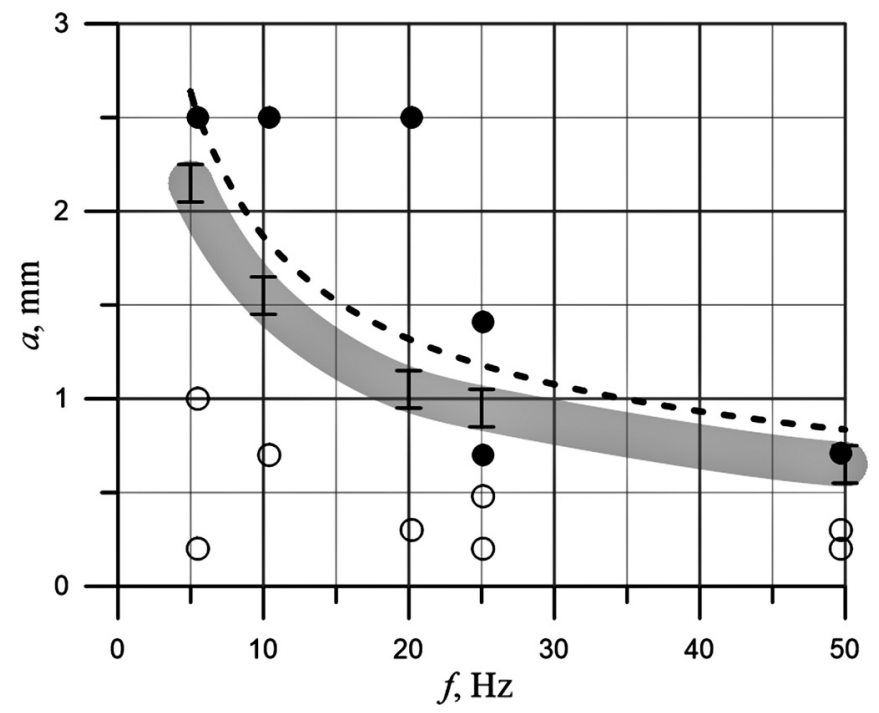

FIG. 10. Stability map for experimental, analytical, and numerical results. Circles are experimental results (open circles: stable states; filled circles: Faraday instability). The gray line shows numerical data (the linewidth corresponds to the accuracy of the stability boundary determination). The dashed line corresponds to analytical stability estimation given by Eq. (36). 


\section{CONCLUSIONS}

In the experimental studies performed with near critical fluid under zero gravity or liquid mixtures, it was shown that under vibrations a periodical pattern (bands) can develop in the system $[5,13,14]$. Depending on the parameters of the vibrations, an instability can be observed in the bands, which leads to the development of Faraday waves. In the present study, such Faraday instabilities in a thin band pattern have been studied taking into account the interaction between the bands. This is a unique situation as compared with the classical Faraday instability in a two-layer system with a single interface or in a periodical pattern of thick bands. An analytical solution has been obtained under assumptions of low viscosity and low surface tension as, e.g., encountered with liquid-vapor interfaces near a critical point. It is shown that the critical vibration amplitude is subjected to a sharp growth for small spatial periods of the band pattern.

A 2D numerical modeling of Faraday waves has been performed using the full Navier-Stokes equations and the volume of fluid (VoF) method for the interface tracking. The influence of vibrations is taken into account by introducing a periodically varying volumetric force. The calculations show that at small spatial periods of band patterns the interaction between the interfaces leads to the significant stabilization of the system. The dynamics of the Faraday waves on the bands corresponds to subharmonic oscillations, i.e., the waves are similar to classical Faraday waves.

The comparison of analytical results on the critical vibration amplitude with the stability curves obtained in the direct numerical simulation and experimental data show good agreement. Nevertheless, a small overestimation of the critical vibration amplitude given by analytical analysis takes place, which was expected since similar deviation was observed by others [14] for a single fluid interface.

\section{ACKNOWLEDGMENTS}

D.B., Y.G., and C.L. acknowledge partial funding support by Centre National d'Etudes Spatiales (CNES). They also thank the European Space Agency (ESA) for its support in sounding rocket experiments. The theoretical analysis and numerical calculations were supported by Russian Foundation for Basic Research (Grant No. 15-01-09069).

[1] G.-H. Wolf, The dynamic stabilization of the Rayleigh-Taylor Instability and the corresponding dynamic equilibrium, Zeitschrift Für Phys. 227, 291 (1969).

[2] D. V. Lyubimov and A. A. Cherepanov, Development of a steady relief at the interface of fluids in a vibrational field, Fluid Dyn. 21, 849 (1986).

[3] D. V. Lyubimov, G. L. Khilko, A. O. Ivantsov, and T. P. Lyubimova, Viscosity effect on the longwave instability of a fluid interface subjected to horizontal vibrations, J. Fluid Mech. 814, 24 (2017).

[4] D. V. Lyubimov, T. P. Lyubimova, and A. A. Cherepanov, Dynamics of Interfaces in Vibration Fields (FizMatLit, Moscow, 2003), in Russian.

[5] T. Lyubimova, A. Ivantsov, Y. Garrabos, C. Lecoutre, G. Gandikota, and D. Beysens, Band instability in near-critical fluids subjected to vibration under weightlessness, Phys. Rev. E 95, 013105 (2017).

[6] D. V. Lyubimov, A. O. Ivantsov, T. P. Lyubimova, and G. L. Khilko, Numerical modeling of frozen wave instability in fluids with high viscosity contrast, Fluid Dyn. Res. 48, 061415 (2016).

[7] E. Talib, S. Jalikop, and A. Juel, The influence of viscosity on the frozen wave instability: theory and experiment, J. Fluid Mech. 584, 45 (2007).

[8] E. Talib and A. Juel, Instability of a viscous interface under horizontal oscillation, Phys. Fluids 19, 092102 (2007).

[9] D. Beysens, R. Wunenburger, C. Chabot, and Y. Garrabos, Effect of oscillatory accelerations on two-phase fluids, Microgravity Sci. Technol. 11, 113 (1998). 
[10] D. Beysens, Y. Garrabos, D. Chatain, and P. Evesque, Phase transition under forced vibrations in critical $\mathrm{CO}_{2}$, EPL 86, 16003 (2009).

[11] D. Beysens, D. Chatain, Y. Garrabos, C. Lecoutre, F. Palencia, P. Evesque, and V. Nikolayev, The effect of vibrations on heterogeneous fluids: Some studies in weightlessness, Acta Astronaut. 61, 1002 (2007).

[12] G. Gandikota, D. Chatain, S. Amiroudine, T. Lyubimova, and D. Beysens, Faraday instability in a nearcritical fluid under weightlessness, Phys. Rev. E 89, 013022 (2014).

[13] P. Salgado Sánchez, V. Yasnou, Y. Gaponenko, A. Mialdun, J. Porter, and V. Shevtsova, Interfacial phenomena in immiscible liquids subjected to vibrations in microgravity, J. Fluid Mech. 865, 850 (2019).

[14] V. Shevtsova, Y. Gaponenko, V. Yasnou, A. Mialdun, and A. Nepomnyashchy, Two-scale wave patterns on a periodically excited miscible liquid-liquid interface, J. Fluid Mech. 795, 409 (2016).

[15] D. V. Lyubimov, A. A. Cherepanov, T. P. Lyubimova, and B. Roux, Orienting effect of vibrations on the interphase, C. R. Acad. Sci. 325, 391 (1997).

[16] H. E. Stanley, Introduction to Phase Transitions and Critical Phenomena (Oxford University Press, Oxford, 1987).

[17] B. Zappoli, D. Beysens, and Y. Garrabos, Heat Transfers and Related Effects in Supercritical Fluids (Springer, Dordrecht, 2015).

[18] Y. Garrabos, C. Lecoutre, S. Marre, D. Beysens, and I. Hahn, Liquid-vapor rectilinear diameter revisited, Phys. Rev. E 97, 020101(R) (2018).

[19] D. V. Lyubimov, T. P. Lyubimova, S. Meradji, B. Roux, D. Beysens, Y. Garrabos, and D. Chatain, 2D unsteady motion and deformation of a gaseous bubble in a vibrating liquid at zero gravity, J. Phys. IV 11, Pr6-91 (2001).

[20] D. V. Lyubimov, A. A. Cherepanov, T. P. Lyubimova, and B. Roux, Deformation of gas or drop inclusion in high frequency vibrational field, Microgravity Q. 6, 69 (1996).

[21] S. Fauve, K. Kumar, C. Laroche, D. Beysens, and Y. Garrabos, Parametric-Instability of a Liquid-Vapor Interface Close to the Critical-Point, Phys. Rev. Lett. 68, 3160 (1992).

[22] K. Kumar and L. S. Tuckerman, Parametric instability of the interface between two fluids, J. Fluid Mech. 279, 49 (1994). 